\title{
Management decision-aiding with a fuzzy integral based Performance Measurement System
}

\author{
L. Berrah ${ }^{1}$, J. Montmain ${ }^{2}$, V. Clivillé ${ }^{1}$, G. Mauris ${ }^{1}$ \\ ${ }^{1}$ LISTIC Université de Savoie \\ ${ }^{2}$ LGI2P Ecole de Mines d'Alès
}

\begin{abstract}
This article is a contribution to the decision-maker's requirements to adapt resource allocation and define rewards according to the performance assessments at each milestone of an improvement action plan execution. Our approach is based on Performance Measurement Systems relating interacting elementary performance to the overall performance by a 2 -additive Choquet integral aggregation. Performance improvement analysis for resource allocation and motivation management is based on the marginal contributions of each elementary performance to the overall performance which give useful information on the way the overall performance has been achieved and would be improved. The propositions are applied to the business turn-over performance improvement of a SME company manufacturing kitchens and bathrooms.
\end{abstract}

Keywords: industrial performance, multicriteria decision-making, Choquet fuzzy integral, management strategies, business turn-over improvement.

\section{Introduction - Problem statement}

In the current context of financial crisis and economic globalisation, manufacturing companies are becoming increasingly concerned with the multicriteria nature of their process assessments [1][2]. Namely, beyond the Taylorian productivity, industrial processes must satisfy numerous and diversified criteria such as delay and quality, but also innovation, environmental and social responsibility criteria [3][4]. In this context, the continuous performance improvement approaches need a strong synergy between the company's strategy, the defined objectives and the launched actions to be carried out in a relevant way. This is the purpose of improvement philosophies such as the Kaizen [5][6], the Lean Manufacturing [7][8], the 6 Sigma [9] ones...

Besides, when the improvement approaches concern structural objectives, that are associated with well controlled action plans, the process is rather easy to manage. But when these approaches are defined for complex and conjunctural objectives, they are subjected to uncertain context evolution. Project managers have thus to adjust their decisions at each step of the improvement process. These decisions generally focus on the validation of reached states, on the allocation of additional resources, and on the reward procedure to motivate the employees.
Generally, the performance improvement is based on the break-down overall performances into elementary ones, and project managers define, for a given temporal horizon, the overall performances to reach. The temporal horizon is also broken-down into a set of milestones that generally correspond to the different evolutions of the associated action plans or to budget control and allocation [10]. At each milestone, based on the overall performance expression as well as the elementary ones, the project manager has to answer the following questions:

- Are the observed intermediary results satisfactory?

- How to allocate the resources for the next period according to the expected final overall performance?

- How to define correct rewards according to the observed results?

Based on our previous proposed model where PMSs were considered for informational control purposes [11][12], the aim of this work is to define pieces of information for aiding the decision-makers to succeed in answering the above questions. The major idea developed here is to model the link between the management strategy and the way in which the overall performance has been achieved. Our approach is based on the determination of marginal contributions of each particular elementary performance to the overall performance issued from a Choquet integral aggregation. Beyond the conventional scorecard that gives the elementary performances, decision-makers get thus well-focused information for the management of the expected overall performance.

This paper is organised as follows. Section II recalls the essential background concerning the performance expression as well as the main project management strategies that we will consider in this study. In section III, the proposed model is described, fundamentally based on the notion of criterion contribution to the performance improvement. Section IV presents an application to a case study submitted by a kitchen manufacturer who wants to continually increase his business turn-over. Some concluding remarks to be considered in the future are finally pointed out. 


\section{Background on performance expression and management strategies}

\subsection{Industrial performance expression background}

From a control point of view, quantified performance expressions are used, on the one hand, to validate the past actions and the achieved states, and, on the other hand, to decide the actions to launch in order to satisfy on the best way the overall objective. However, the quantification mechanism requires a preliminary identification of the performance structure by the break-down of the considered overall objective into elementary ones at different organisational levels (strategic, tactical or operational). Generally, this identification is achieved by expertise and results in logical relationships between the involved performance criteria. Then, the two main sub problems concerning the quantification of the performance are:

- the expression of quantified performances reflecting the satisfaction of the elementary objectives,

- the expression of a quantified performance reflecting the satisfaction of the overall objective knowing that a direct determination is generally not possible.

Concerning the last point, it is closely related to the preferences of the decision maker with respect to the different (often conflicting) objectives. In multiobjectives optimization methods, such as the Pareto front ones, these preferences are required only at the latest stage to select the most appropriate among the Pareto equivalent ones, and thus these preferences have to be defined on a limited numbers of situations [13]. In aggregative methods, the preferences have to be defined first, and this requires a deeper knowledge of the relationships between (often interacting) criteria which can be difficult to quantify. Nevertheless, as recalled hereafter, our works are based on aggregation operators for the synthesis of the elementary performances into an overall performance, and the Macbeth methodology to determine the criteria weights and interaction coefficients.

In the context of industrial performance expression, the aggregation processing can be formalized by the following mapping [12]:

$$
\begin{gathered}
A \mathrm{~g}: \quad E_{1} \times E_{2} \times \ldots E_{n} \rightarrow E \\
\left(p_{1} \ldots p_{\mathrm{i}} \ldots p_{\mathrm{n}}\right) \rightarrow p_{\text {Overall }}=A g\left(p_{1} \ldots p_{\mathrm{i}} \ldots p_{\mathrm{n}}\right)
\end{gathered}
$$

$E_{\mathrm{i}}$ being respectively the sets of the elementary pieces of information $p_{\mathrm{i}}$ and $E$ of the aggregated information denoted $p_{A g}$. Regarding the information involved in the performance indicators, $p_{\mathrm{i}}$ is the elementary performance expression, and $p_{\text {Overall }}$ is the overall performance expression. Determining coherently elementary and overall performance expressions requires mainly two points [14].

- The elementary expressions (according to the different considered criteria) have to be defined on the same scale type, generally along the same interval scale $[0,1]$.

- The aggregation operator must be significant for the considered scale type.

The choice of the aggregation operator is a key point. To deal with the criteria interaction, the family of the Choquet Integral (denoted $C_{\mu}$ ) operators is interesting.

Indeed, the $C_{\mu}$ operators can be seen as an extension of the weighted average mean operators, able to consider mutual interaction between criteria [15]. The $C_{\mu}$ operators are also significant for performance expressions defined on an interval scale [14].

\subsection{The overall performance expression}

In our industrial context, the 2-additive Choquet integral that considers only interactions by pairs has been shown relevant [16]. Two kinds of parameters are involved:

1. the weight of each elementary performance expression in relation to all the other contributions to the overall performance evaluation by the socalled Shapley parameters $v_{\mathrm{i}}{ }^{\prime} s$, that satisfy the condition $\sum_{\mathrm{i}=1}^{\mathrm{n}} v_{\mathrm{i}}=1$, which is a natural condition for decision-makers,

2. the mutual interaction parameters $I_{\mathrm{ij}}$ of any pair of performance criteria, which range within the interval $[-1,1]$.

Thus, the aggregated overall performance by the 2 additive $C_{\mu}$ is given by:

$$
p_{\text {overall }}=C_{\mu}(\vec{P})=\sum_{\mathrm{i}=1}^{\mathrm{n}} \nu_{\mathrm{i}} p_{\mathrm{i}}-\frac{1}{2} \sum_{\mathrm{i}=1}^{\mathrm{n}} I_{\mathrm{ij}}\left|p_{\mathrm{i}}-p_{\mathrm{j}}\right|
$$

where $\vec{P}=\left(p_{1} \ldots, p_{\mathrm{i}} \ldots, p_{\mathrm{n}}\right)$ is the elementary expressions vector with the property:

$$
\left(v_{\mathrm{i}}-\frac{1}{2} \sum_{\mathrm{j}=1}^{\mathrm{n}}\left|I_{\mathrm{ij}}\right|\right) \geq 0, \forall i \in[1, n], j \neq i
$$

The main problem on the practical level is to dispose of a procedure to determine these aggregation parameters from the qualitative manager knowledge. In this aim, we have applied the MACBETH methodology [17] which ensures the coherence of the performance expression on an interval scale, and also provides a convenient manager asking procedure [11]. Therefore by applying the MACBETH procedure, the manager can design a PMS providing both elementary and overall performance expressions. These pieces of information are useful for assessment purposes, but are not sufficient to efficiently manage the performance improvement cycles as explained below. 


\subsection{Management strategies for performance im- provement}

In fact, in performance improvement on a quite long temporal horizon, project managers can adopt many attitudes with regard to the decisions to be made at each milestone. On the one hand, the decision making depends on the organizational structure of the control used by the company [18]. The decision-maker behaviour depends also on social choice aspects. In particular the problem of allocating resources or rewards to the different actors of elementary performance improvement should consider motivation aspects in addition to technical ones.

In the sociological and economic literature, this problem relates to notions of distributive justice, more specifically desert-based principles of distribution [19].

- People should be rewarded according to the value of their contribution to the final product.

- People should be rewarded according to the effort they expend in their work activity.

- Resource allocation can compensate for difficulty differences in people's work activities.

To deal with all these principles, the manager can consider more or less utilitarian or egalitarian strategies as will be illustrated in section 3 .

Note finally that the decision-maker behaviour depends also on the psychological aspects related to the affect notion [20], and eventually on the points of view of other people in case of group decision-making. Both these aspects are not considered in this paper.

\section{Performance improvement management}

Let us recall that we consider a context where project managers define, for a given temporal horizon, the overall performances to be achieved. The temporal horizon is also broken-down into a set of milestones that generally correspond to the different evolutions of the associated action plans or to budget control and allocation. At each milestone, based on the overall performance value as well as the elementary ones, the project manager has to answer the following questions.

- Are the observed intermediary results satisfactory and possibly how to define correct rewards?

- How to allocate the resources for the next period according to the expected final overall performance?

These points can be translated into the following mathematical statements.

- What has been at the considered milestone the marginal contribution of one particular elementary performance to overall performance?

- What would be the expected marginal contribution of one particular elementary performance to the overall performance during the remaining periods?

\subsection{2-additive Choquet integral expression}

As presented in section 2, the Choquet integral is composed of a linear part that is modified according to mutual interactions between criteria. Going beyond this, the 2-additive Choquet integral can be written as a conventional weighted mean in each simplex $H_{\sigma_{k}}=\left\{x \in[0,1] / 0 \leq p_{\sigma(1)}^{k} \leq \ldots \leq p_{\sigma(n)}^{k} \leq 1\right\} \quad$ defined by the ranking of the elementary performances represented by the function $\sigma$; this weighted sum can be simply expressed with the Shapley and interactions indices [21]. Indeed, for any $\vec{P}^{k}$ performance vector belonging to the $H_{\sigma_{k}}$ simplex, the Choquet integral has the linear expression:

$$
\begin{gathered}
p_{\text {overall }}^{k}=C_{\mu}\left(\vec{P}^{k}\right)=\sum_{i=1}^{n} \Delta \mu_{\sigma(i)}^{k} \cdot p_{\sigma(i)}^{k} \text {, where } \\
\Delta \mu_{\sigma(i)}^{k}=v_{\sigma(i)}+\frac{1}{2} \sum_{j>i} I_{\sigma(i) \sigma(j)}-\frac{1}{2} \sum_{j<i} I_{\sigma(j) \sigma(i)} \text { with } v_{\sigma(i)} \text {-the }
\end{gathered}
$$
relative importance of criterion $c_{\sigma(i)}$ and $I_{\sigma(i) \sigma(j)}$ the interaction between criteria $c_{\sigma(i)}$ and $c_{\sigma(j)}$. Moreover, for every $k: \sum_{i=1}^{n} \Delta \mu_{\sigma(i)}^{k}=1$ Thus, the Choquet integral is a type of weighted mean with weights varying according to the ranking of elementary performances. Therefore, mathematical tools developed in the linear framework can be extended, as detailed below.

\subsection{A posteriori contributions}

Our approach consists in rewriting the performance improvement, i.e. $C_{\mu}\left(\vec{P}^{*}\right)-C_{\mu}\left(\vec{P}^{I}\right)$ from an initial performance vector $\vec{P}^{I}$ to a final performance vector $\vec{P}^{*}$, as a sum of terms related to the elementary performances. Actually, elementary performance contributions depend on the path followed during the performance improvement. For example, to increase the performance from $\vec{P}^{I}$ to $\vec{P}^{*}$ three possible paths $t_{j=1,2 \text { et } 3}$ can be considered as illustrated in the figure 1: with $t_{1}, P_{1}$ is first increase then $P_{2}$, for $t_{2}$ it is the opposite, and for $t_{3}, P_{1}$ is half increase, then $P_{2}$ and finally $P_{1}$ again.

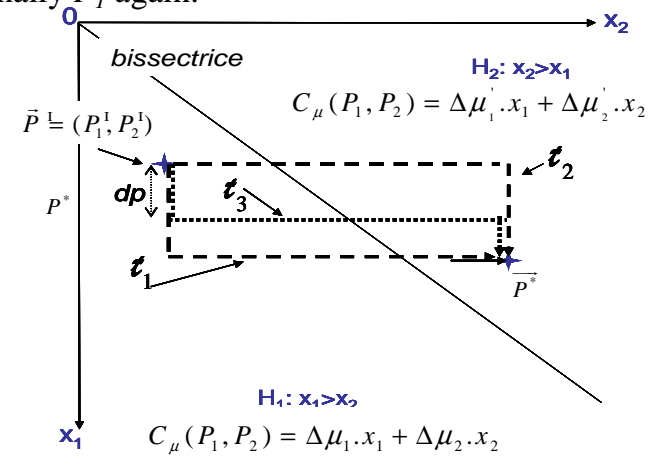

Fig. 1: Contributions according to improvement paths 
An important fact is that the criteria contributions to $C_{\mu}\left(\vec{P}^{*}\right)-C_{\mu}\left(\vec{P}^{I}\right)$ depend on the individual path. The contributions of criterion 1 according to the different paths $t_{i=1,2 \text { et } 3}, \quad C_{1}^{t_{j}} \quad$ are respectively: $C_{1}^{t_{1}}=\Delta \mu_{1} \cdot\left(p_{1}^{*}-p_{1}^{I}\right), \quad C_{1}^{t_{2}}=\Delta \mu_{1}^{\prime} \cdot\left(p_{1}^{*}-p_{1}^{I}\right) \quad\left(\Delta \mu_{1}\right.$ and $\Delta \mu_{1}^{\prime}$ are the weights in the $\mathrm{H} 1$ and $\mathrm{H} 2$ simplexes) and $C_{1}^{t_{3}}=\Delta \mu_{1} \cdot d p+\Delta \mu_{1}^{\prime} \cdot\left(p_{1}^{*}-p_{1}^{I}-d p\right)$. Similarly, for the criterion 2:

$C_{2}^{t_{1}}=\Delta \mu_{2} \cdot\left(p_{1}^{I}-p_{2}^{I}\right)+\Delta \mu_{2}^{\prime} \cdot\left(p_{2}^{*}-p_{1}^{I}\right)$,

$C_{2}^{t_{2}}=\Delta \mu_{2} \cdot\left(p_{1}^{I}-p_{2}^{I}\right)+\Delta \mu_{2}^{\prime} \cdot\left(p_{2}^{*}-p_{1}^{I}\right)$ and

$C_{2}^{t_{3}}=\Delta \mu_{2} \cdot\left(p_{1}^{I}+d p-p_{2}^{I}\right)+\Delta \mu_{2}^{\prime} \cdot\left(p_{2}^{*}-p_{1}^{I}-d p\right)$.

It can easily be checked that:

$\forall j, C_{\mu}\left(\vec{P}^{*}\right)-C_{\mu}\left(\vec{P}^{I}\right)=C_{1}^{t_{j}}+C_{2}^{t_{j}}$

with $\Delta \mu_{1}+\Delta \mu_{2}=\Delta \mu_{1}^{\prime}+\Delta \mu_{2}^{\prime}=1$ (i.e. Choquet integral continuity).

Consequently, the a posteriori contribution of criterion $C_{i}^{t_{j}}$ can easily be computed knowing the followed path $\mathrm{t}_{\mathrm{j}}$.

\subsection{A priori contributions}

But, as a consequence of our modelling point of view, the a priori contribution of a criterion to $C_{\mu}\left(\vec{P}^{*}\right)-C_{\mu}\left(\vec{P}^{I}\right)$ cannot be precisely determined.

Thus, we propose to compute the lower and upper endpoints of the interval containing all possible values for the contribution of any criterion $i$. The aim here is to provide both the minimum and maximum expected contributions for each elementary performance. Indeed, one criterion performance improvement must necessarily contribute at least up to $C_{i}^{N}=\min C_{i}^{t}$, but it is entirely possible for the contribution to reach $C_{i}^{\Pi}=\max _{t} C_{i}^{t}$. Therefore, $\left[C_{i}^{N}, C_{i}^{\Pi}\right]$ characterises the imprecision in the a priori contribution of criterion $i$ to the overall performance improvement $C_{\mu}\left(\vec{P}^{*}\right)-C_{\mu}\left(\vec{P}^{I}\right)$.

The principle used to compute $\left[C_{i}^{N}, C_{i}^{\Pi}\right]$ involves a three-step procedure.

Step 1: First, let us consider the non-oriented complete graph $\Gamma$, which ties all the $n !$ simplexes $H_{\sigma}=\left\{\vec{P} \in[0,1]^{n} / 0 \leq p_{\sigma(1)} \leq . . \leq p_{\sigma(n)} \leq 1\right\}$. Let $H_{\sigma_{I}}$ be the simplex the initial performance vector $\vec{P}^{I}$ is belonging to and let $H_{\sigma_{F}}$ be the simplex of the final performance vector $\vec{P}^{*}$. Thus, $H_{\sigma_{I}}$ would be the source of $\Gamma$ and $H_{\sigma_{F}}$ the sink.

- For each $H_{\sigma}$, it is verified that at least one point $\vec{P}(\sigma)$ exists such as: $\forall i \in\{1 ; . . ; n\}, \quad p_{i}^{I} \leq p_{i}(\sigma) \leq p_{i}^{*} ;$
- If such a point does not exist, then $H_{\sigma}$ is to be deleted along with all arcs whose $H_{\sigma}$ is an endpoint;

- Lastly, once each node $H_{\sigma}$ has been verified, a filtered graph $\Gamma_{\mathrm{F}}$ is obtained.

Step 2: For each node $H_{\sigma}$ in $\Gamma_{\mathrm{F}}$, the range of allowed values for $p_{i}(\sigma)$ is computed for each criterion $i$ : $\left[B_{i}^{\operatorname{lnf}}(\sigma) ; B_{i}^{\text {Sup }}(\sigma)\right]$. This computation is given by the following expression:

$$
B_{i}^{\text {Inf }}(\sigma)=\max _{j \leq \sigma^{-1}(i)} p_{\sigma(j)}^{I} ; B_{i}^{S u p}(\sigma)=\min _{j \geq \sigma^{-1}(i)} p_{\sigma(j)}^{*}
$$

An example is given in figure 2 for $i=4$ when $n=4$ and $H_{\sigma}=\left\{x \in[0,1] / 0 \leq p_{1} \leq p_{4} \leq p_{2} \leq p_{3} \leq 1\right\}$. Then, figure 2 illustrates the $\left[B_{4}^{l n f}(\sigma) ; B_{4}^{S u p}(\sigma)\right]$ computation.

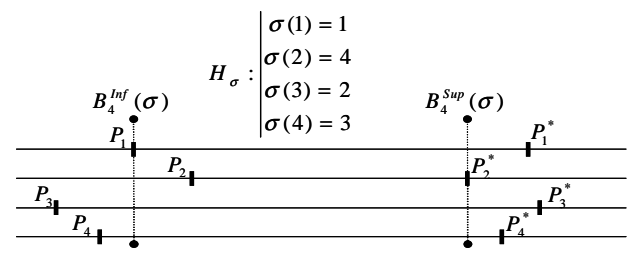

Fig. 2 - Example of $\left[B_{i}^{\text {hff }}(\sigma) ; B_{i}^{\text {Sup }}(\sigma)\right]$ computation

Step 3: $\Gamma_{\mathrm{F}}$ defines a set of paths Path $_{k}, k=1 . . m$, with $H_{\sigma_{I}}$ as the source and $H_{\sigma_{F}}$ the sink without the presence of any cycles. For each node $H_{\sigma}$ in $\mathrm{Path}_{k}$, a determination of $\left[B_{i}^{\text {Inf }}(\sigma) ; B_{i}^{\text {Sup }}(\sigma)\right]$ and $\Delta \mu_{i}(\sigma)$ can be made in addition to the linear coefficient of $C_{\mu}$ for the criterion $i$ in the simplex $H_{\sigma}$. Let $\varepsilon_{i}^{\text {Path }_{k}}$ be the series of disjoint intervals $I_{i}(\sigma)$ such as $I_{i}(\sigma) \subseteq\left[B_{i}^{\operatorname{lnf}}(\sigma) ; B_{i}^{\text {Sup }}(\sigma)\right]$ and $\bigcup_{\sigma / H_{\sigma} \in \text { Nodes }\left(\text { Path }_{k}\right)} I_{i}(\sigma)=\left[p_{i}^{I} ; p_{i}^{*}\right]$. With $L(I)$ denoting the length of interval $I$, the following expressions can then be computed:

$$
\begin{aligned}
& \min _{\varepsilon_{c}^{\text {pant }}} \sum_{\sigma / H_{\sigma} \in \text { Nodes }\left(\text { Path }_{k}\right)} \Delta \mu_{i}(\sigma) \cdot L\left(I_{i}(\sigma)\right) \text { and } \\
& \max _{\varepsilon_{c}^{p a w_{k}}} \sum_{\sigma / H_{\sigma} \in \text { Nodes }\left(\text { Path }_{k}\right)} \Delta \mu_{i}(\sigma) \cdot L\left(I_{i}(\sigma)\right)
\end{aligned}
$$

Ultimately:

$$
\begin{aligned}
& C_{i}^{N}=\min _{\text {Path }_{k}} \min _{\varepsilon_{i}^{\text {Path }_{k}}} \sum_{\sigma / H_{\sigma} \in \text { Nodes }\left(\text { Path }_{k}\right)} \Delta \mu_{i}(\sigma) \cdot L\left(I_{i}(\sigma)\right) \\
& C_{i}^{\Pi}=\max _{\text {Path }_{k}} \max _{\varepsilon_{i}^{\text {Puth }_{k}}} \sum_{\sigma / H_{\sigma} \in \text { Nodes }\left(\text { Path }_{k}\right)} \Delta \mu_{i}(\sigma) \cdot L\left(I_{i}(\sigma)\right)
\end{aligned}
$$

These contribution intervals enable to quantitatively compare the monitoring strategies to improve the overall performance from $\vec{P}^{I}$ to $\vec{P}^{*}$. 


\subsection{Monitoring the performance improvement}

For performance improvement monitoring purposes, $C_{i}^{N}$ and $C_{i}^{\Pi}$ can be computed at each milestone point $\vec{P}^{k}$ of the performance evolution from $\vec{P}^{I}$ to $\vec{P}^{*}$. The corresponding $\left[C_{i}^{N}(k), C_{i}^{\Pi}(k)\right]$ intervals satisfy the following relationship:

$\left[C_{i}^{N}(k), C_{i}^{\Pi}(k)\right] \supseteq\left[\hat{C}_{i}(k)+C_{i}^{N}(k+1), \hat{C}_{i}(k)+C_{i}^{\Pi}(k+1)\right]$ where $\hat{C}_{i}(k)$ is the a posteriori contribution at milestone $k$ and $C_{i}^{N}(k)\left(\right.$ resp. $\left.C_{i}^{\Pi}(k)\right)$ the lowest (resp. highest) expected contribution from milestone $\vec{P}^{k}$ to $\vec{P}^{*}$. This means that the imprecision related to the contribution of a criterion to the overall final performance improvement $C_{\mu}\left(\vec{P}^{*}\right)-C_{\mu}\left(\vec{P}^{I}\right)$ naturally decreases as $\vec{P}^{k}$ approaches $\vec{P}^{*}$. Indeed, once $\vec{P}^{*}$ has been reached at $k=k^{*}, C_{i}^{N}\left(k^{*}\right)=C_{i}^{\Pi}\left(k^{*}\right)$, no imprecision remains.

The manager thus knows at any milestone the full range of expected values for each criterion contribution, but he could use these pieces of information in different ways, according to his management strategy, for resources and rewards sharing.

Hereafter we illustrate a few possible strategies without entering into the debate of which one would be the best. We consider a situation where the final performance to be expected at the final time is $P^{*}=(1,1, \ldots, 1)$ and where several milestones (indexed $k$ ) have been defined at the beginning. Let us denote $A m$ the total resources amount granted for the considered performance improvement (for ease of processing the resources are transformed into monetary units (a corresponding coefficient $\alpha(k)$ is introduced in the following) though in practice they correspond to equipment or workers). The reward $R w$ corresponds to the total amount dedicated to reward efforts during the improvement duration. Hereafter, without being exhaustive we describe two strategies considered as possible by our industrial partner.

\section{Monitoring Strategy 1}

The manager wants to reward the employee teams in charge of criterion performance improvements according to each elementary performance expected value without creating competition between them. The only condition for $A m$ to be shared fairly is that all the elementary objectives has been fully achieved.

The corresponding procedure is:

$k=0, \forall i, \beta_{i}^{0}=0$

While $k<k^{*}$

Allocate $A m$ to $\alpha(k) \cdot\left[p_{i}^{*}-p_{i}^{0}\right], \alpha(k) \in[0,1]$ is a time decreasing function such as $\sum_{k} \alpha(k)=1$

$k=k+1$

At each milestone $\vec{P}(k)$, the $p_{i}^{k}$ observed value is such that: $p_{i}^{k}=\left(1-\beta_{i}^{k}\right) \cdot p_{i}^{0}+\beta_{i}^{k} \cdot p_{i}^{*}$
Share rewards amount $R w$ proportionally to

$$
\begin{aligned}
& \left(\beta_{i}^{k}-\beta_{i}^{k-1}\right) \cdot \frac{R w}{n} \\
& \left.p_{i}^{k}-p_{i}^{k-1}=\left(\beta_{i}^{k}-\beta_{i}^{k-1}\right) \cdot\left(p_{i}^{*}-p_{i}^{0}\right)\right)
\end{aligned}
$$

(namely,

end while

\section{Monitoring Strategy 2}

The manager prefers to first consider criteria that should guaranty the most significant overall performance increase. Rewards are then related to the criteria contributions to the overall performance improvement. Such a share corresponds to a cautious strategy of the company without further considerations regarding the social climate. Indeed, a criterion contribution depends on the simultaneous efforts of others and this induces competition between criteria because of the uncertainty aspect of the improvement progress.

The corresponding procedure is:

$k=0, \forall i, \hat{C}_{i}^{0}=0$

While $k<k^{*}$

Allocate resources $A m$ to $\alpha(k)$. [ $\left[C_{i}^{N}(k), C_{i}^{\Pi}(k)\right]$ (here as a precise value is required different possibilities can be considered: for example take $C_{i}^{N}(k)$ or $C_{i}^{\Pi}(k)$, or the middle of them)

$k=k+1$

At each milestone $\vec{P}(k)$, compute the corresponding a posteriori contributions $\hat{C}_{i}(k)$ (see subsection 3.2)

Share rewards amount $R w$ proportionally to $\frac{\hat{C}_{i}(k)-\hat{C}_{i}(k-1)}{C_{\mu}\left(\vec{P}^{*}\right)-C_{\mu}\left(\vec{P}^{I}\right)} \cdot R w$

(namely, $\frac{R w}{C_{\mu}\left(\vec{P}^{*}\right)-C_{\mu}\left(\vec{P}^{I}\right)} \cdot \sum_{i} \sum_{k}\left(\hat{C}_{i}(k)-\hat{C}_{i}(k-1)\right.$ )

$\left.=\frac{R w}{C_{\mu}\left(\vec{P}^{*}\right)-C_{\mu}\left(\vec{P}^{I}\right)} \cdot \sum_{i} \hat{C}_{i}\left(k^{*}\right)=R w\right)$

end while

\section{Case study}

\subsection{Presentation}

The case study is issued from the Fournier Company, a SME that produces kitchens, bathrooms and storing closets. The company manufactures more than 850000 items and its weekly production is 5000 pieces of furniture. The product is made of about 10 different parts, each part containing between 10 and 50 elementary pieces. The manufacturing cycle time is between 2 and 5 weeks. The goal of the company is to continuously increase its business turn-over by about 10\% per year. More particularly, since the beginning of the financial crisis in 2008, the company has focused on the management system of the retailers' network. Indeed, in the previous period, the major objective was associated to the productivity and the performance of the factory. It is 
necessary today for the company to consider the indirect activities, such as logistics, marketing, advertising... without forgetting the industrial criteria of cost, quality and delay.

In this new context for the Fournier Company, top management has expressed its needs for formal tools for the monitoring and the planning of the improvement approach. In this sense it is proposed for the company to use the previous PMSs to supply on the one hand the performance expressions for the duration of the improvement, and on the second hand, the information about the past contribution and the expected ones at each milestone of the improvement project.

\subsection{The company PMS}

Knowing the overall objective of Business turn-over increase of $10 \%$ per year; the objective breakdown allows the top management to define four elementary objectives (as shown in figure 3 ) which were yet achieved (table 1) at the beginning of the year 2010.

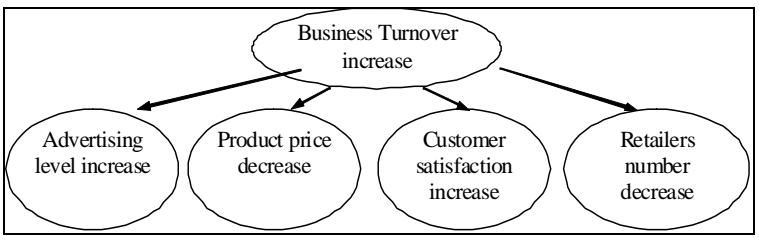

Fig. 3: The Business turn-over objective breakdown

The current situation (in the beginning of the year 2010 is gathered in the table 1.

\begin{tabular}{|c|c|c|}
\hline Objective label & $\begin{array}{l}\text { Objective } \\
\text { value (July } \\
2011)\end{array}$ & $\begin{array}{l}\text { Current } \\
\text { value (be- } \\
\text { ginning of } \\
2010)\end{array}$ \\
\hline Advertising budget & 10 & 6 \\
Product price & 10 & 12.5 \\
Customer satisfaction & $98 \%$ & $93,2 \%$ \\
New retailers number & $50 /$ year & $10 /$ year \\
\hline
\end{tabular}

Table 1: Current state and objective quantification

From this information and thanks to the project manager expertise it is possible to build the company PMS (cf. 2.2), i.e. to identify:

- the elementary performance expressions according to the previous objective breakdown,

- the overall one by the determination of the parameters of the $C_{\mu}$ operator (table 1).

\begin{tabular}{|l|l|l|l|l|}
\hline Shapley Indexes & $\mathrm{v}_{1}$ & $\mathrm{v}_{2}$ & $\mathrm{v}_{3}$ & $\mathrm{v}_{4}$ \\
\hline & 0.30 & 0.25 & 0.30 & 0.15 \\
\hline Interactions & $\mathrm{I}_{12}$ & $\mathrm{I}_{13}$ & $\mathrm{I}_{34}$ & \\
\hline & 0.30 & 0.20 & 0.25 & \\
\hline
\end{tabular}

Table 1: $C_{\mu}$ parameters

The elementary performances and the $C_{\mu}$ parameters have been obtained by applying the Macbeth procedure that requires the decision-makers to give his intensities of preference about a set of situations. The decisionmaker was already familiar with the comparison of situations and the answers came rapidly with the help of the research team. Nevertheless the time dedicated to this phase (one afternoon in two consecutive weeks) does not make it possible frequently executing this procedure.

Thus, the initial elementary performances are $\vec{P}^{I}=(0.8,0.2,0.75,0.5)$ with $p_{\text {Overall }}^{I}=0.4638$. The expected performance for the end of 2011 is $\vec{P}^{*}=(1,1,1,1)$ with $p_{\text {Overall }}^{*}=1$, i.e. the objectives must be achieved. The total resource amount of the project is $A m=460 \mathrm{k} €$.

The total reward amount $R w$ dedicated to employee's efforts during the improvement duration is $500 \mathrm{k} €$.

The company has considered that the second monitoring strategy (see section 3.4) corresponds to his usual management strategy. Indeed for a reactive and efficient control of the improvement project, the manager would like:

- to know the performances at the considered milestone,

- to define right rewards according to the observed results,

- to allocate the resources for the next period according to the expected final overall performance.

\subsection{Improvement strategy deployment}

The manager proposes to define a milestone every semester during the carrying out of the improvement project: $k \in\{1,2,3\}$. He decides also to balance the amount $A m=460 k €$ for resource allocation on the three semesters. He performs in a similar way for the rewards amount $R w=500 k €$. Let us consider the allocation resources and the rewards determination according to the monitoring strategy 2 (see section 3.4)

\section{Beginning of the first semester: $\mathrm{k}=0$}

At the beginning of $2010, \vec{P}^{I}=(0.8,0.2,0.75,0.5)$ with $p_{\text {Overall }}^{I}=0.4638$ and the PQM takes $\alpha(0)=0.5$ (50\% of the resource are affected to the first semester), and the mean contribution is considered for the resource allocation.

The contribution results are gathered in Table 3.

\begin{tabular}{|l|l|l|l|l|}
\hline & $\mathrm{p}_{1}$ & $\mathrm{p}_{2}$ & $\mathrm{p}_{3}$ & $\mathrm{p}_{4}$ \\
\hline$C_{i}^{N}(k=0)$ & 0.029 & 0.26 & 0.01 & 0.075 \\
\hline$C_{i}^{\Pi}(k=0)$ & 0.131 & 0.32 & 0.11 & 0.138 \\
\hline$C_{i}^{\text {mean }}(k=0)=\left(C_{i}^{N}+C_{i}^{\Pi}\right) / 2$ & 0.08 & 0.29 & 0.06 & 0.1065 \\
\hline$\left[1-p_{i}^{0}\right]$ & 0.2 & 0.8 & 0.25 & 0.5 \\
\hline
\end{tabular}

Table 3: first semester a priori analysis 
According to the results of table 3 , the elementary performance $p^{2}$ appears as a priority in the improvement project. The manager then decides to share resources according to the monitoring strategy 2 .

The weighted monetary transformation parameter $c_{w}$ is computed thanks to:

$c_{W} \cdot(0.08+0.29+0.06+0.1065)=0.5 .460 \Rightarrow c_{W}=428.7$.

Then allocated resources are:

428.7. $(0.08,0.29,0.06,0.1065)=(34.3,124.3,25.7,45.7) \mathrm{k} €$.

Note that if monitoring strategy 1 is preferred (resources allocation is independent of the relative importance of criteria), then the normalized monetary transformation parameter is $c .(0.2+0.8+0.25+0.5)=230$ $\Rightarrow c=131.4$. The resource allocation would be: 131.4. $(0.2,0.8,0.25,0.5)=(26.3,105.1,32.9,65.7)$.

\section{Beginning of the first semester: $\mathrm{k}=1$}

Thanks to the effort and actions made during the first semester, the performances given in table 4 have been achieved. At this milestone, the manager gets the elementary performance contributions and determines the associate rewards according to the monitoring strategy 2 (see table 4).

\begin{tabular}{|l|l|l|l|l|}
\hline & $\mathrm{p}_{1}$ & $\mathrm{p}_{2}$ & $\mathrm{p}_{3}$ & $\mathrm{p}_{4}$ \\
\hline$p_{i}(k=1)$ & 0.859 & 0.859 & 0.859 & 0.5 \\
\hline$\hat{C}_{i}(k=1)$ & 0.03 & 0.264 & 0.003 & 0.0 \\
\hline$\frac{R w}{1-C_{\mu}\left(\vec{P}^{I}\right)} \cdot\left[\hat{C}_{i}(k=1)\right]$ & 28.0 & 246 & 2.8 & 0.0 \\
\hline
\end{tabular}

Table 4: first semester a posteriori analysis

Note that $\vec{P}^{1}=(0.859,0.859,0.859,0.5)$ and the overall performance is $C_{\mu}\left(\vec{P}^{1}\right)=0.76$, the global reward amount is $276.8 \mathrm{k} €$ and it remains $230 \mathrm{k} €$ of resources.

At this moment the manager is in similar situation as the beginning of the first semester. He has to determine again the resource allocation for the $2^{\text {nd }}$ semester. Thus, the new contribution intervals for all the criteria $\left[C_{i}^{N}(k=1), C_{i}^{\Pi}(k=1)\right]$ are computed (see table 5 line 1 and 2). Lines 4 and 5 give the interval of the sum of past contribution and expected ones. The arrows indicate the variations with the expected contributions at $\mathrm{k}=0$ (Table 3).

\begin{tabular}{|l|l|l|l|l|}
\hline & $\mathrm{p}_{1}$ & $\mathrm{p}_{2}$ & $\mathrm{p}_{3}$ & $\mathrm{p}_{4}$ \\
\hline$C_{i}^{N}(k=1)$ & 0.011 & 0.014 & 0.007 & 0.102 \\
\hline$C_{i}^{\Pi}(k=1)$ & 0.074 & 0.056 & 0.078 & 0.138 \\
\hline$\hat{C}_{i}(1)+C_{i}^{N}(1)$ & $0.041 \nearrow$ & $0.278 \nearrow$ & $0.01-$ & $0.102-$ \\
\hline$\hat{C}_{i}(1)+C_{i}^{\Pi}(1)$ & $0.104 \searrow$ & $0.32-$ & $0.081 \searrow$ & $0.138-$ \\
\hline$\left[1-p_{i}(1)\right]$ & 0.141 & 0.141 & 0.141 & 0.5 \\
\hline
\end{tabular}

Table 5: second semester a priori analysis
The expected maximal contribution regarding criterion 1 decreases whereas the minimal expected contribution increases. The expected maximal contribution regarding criterion 2 is steady whereas the minimal expected contribution increases, and so on.

Note also that the imprecision regarding the expected contribution of a criterion decreases in time:

$$
C_{i}^{N}(k=0) \leq \hat{C}_{i}(1)+C_{i}^{N}(1) \leq \hat{C}_{i}(1)+C_{i}^{\Pi}(1) \leq C_{i}^{\Pi}(k=1) .
$$

\section{Beginning of the third semester: $\mathrm{k}=2$}

The same a priori and a posteriori analyses are made and the results are reported in table 6 .

\begin{tabular}{|l|l|l|l|l|}
\hline & $\mathrm{p}_{1}$ & $\mathrm{p}_{2}$ & $\mathrm{p}_{3}$ & $\mathrm{p}_{4}$ \\
\hline$p_{i}(k=2)$ & 1.0 & 1.0 & 1.0 & 0.636 \\
\hline$\hat{C}_{i}(k=2)$ & 0.041 & 0.278 & 0.081 & 0.037 \\
\hline$C_{i}^{N}(k=2)$ & 0.0 & 0.0 & 0.0 & 0.1 \\
\hline$C_{i}^{\Pi}(k=2)$ & 0.0 & 0.0 & 0.0 & 0.1 \\
\hline$\hat{C}_{i}(2)+C_{i}^{N}(2)$ & 0.041 & 0.278 & 0.081 & 0.137 \\
\hline$\hat{C}_{i}(2)+C_{i}^{\Pi}(2)$ & 0.041 & 0.278 & 0.081 & 0.137 \\
\hline$\left[1-p_{i}(k=2)\right]$ & 0.0 & 0.0 & 0.0 & 0.364 \\
\hline$\frac{\hat{C}_{i}(2)-\hat{C}_{i}(1)}{1-C_{\mu}\left(\vec{P}^{I}\right)} . R w$ & 10.3 & 13.1 & 72.7 & 34.5 \\
\hline
\end{tabular}

Table 6: third semester analysis

The overall performance is $C_{\mu}\left(\vec{P}^{2}\right)=0.90$ and the global reward is $130.6 \mathrm{k} €$. Obviously the elementary performance $p^{1}, p^{2}, p^{3}$, can no more contribute to the overall one.

For the third semester $k=3$, the remaining resource amount is $115 \mathrm{k} €$ and the last improvement stage to achieve the expected performance $\vec{P}^{*}=(1,1,1,1)$ concerns $p_{4}$. The final contributions are:

$\hat{C}_{1}(k=3)=0.041, \hat{C}_{2}(k=3)=0.278$,

$\widehat{C}_{3}(k=3)=0.081$ and $\hat{C}_{4}(k=3)=0.107$.

It can be checked that:

$0.041+0.278+0.081+0.137=0.537=1-p^{I}$.

In summary, the resource allocation related with the potential contribution for a given criterion fits well the company management philosophy. Indeed, the manager can globally consider the contribution to the overall performance due to the elementary performance improvement. This contribution is seen as more significant than elementary performance increases. To better adjust his resource allocation, the manager suggests that contribution simulation could be an interesting development.

The effect of the rewards on the performance expressions or contributions is not so clear. If the rewards give an additional motivation to the concerned employees, it 
has an opposite effect on the other employees. This last consideration shows that it is difficult to manage the reward issue only by the contributions. Some adaptation of the management strategy by taking efforts in the work activity should be considered in this sense.

\section{Conclusion}

This paper proposes decision aiding pieces of information to better monitor performance improvement when decision-makers are faced with interacting multicriteria modeled by a 2 -addtive Choquet integral. The approach is based on the determination of a posteriori criterion contribution and a priori minimal and maximal expected criterion contribution. Thus the designed Performance Measurement Systems is an aid for management decisions concerning allocation resources and rewards distribution, as illustrated in the case submitted by a SME company.

Here only a few monitoring strategies have been considered. Integrating contribution, effort and cost in different manners for resource allocation and rewards distribution constitutes interesting perspectives. An extension of the method could also be to define elementary objectives for each employee, and to check their contribution to the elementary then overall objectives. In that case, it is interesting to notice that the rewards could take into account the satisfaction of dependent objectives by other employees, i.e. the principle could be that an employee is rewarded if he has done his best according to what is possible, even if the result is depending on the satisfaction of the influencing objectives.

\section{References}

[1] L. Berrah, G. Mauris, L. Foulloy and A. Haurat, Global vision and performance indicators for an industrial improvement approach, Computers in Industry, 43: 211-225, 2000.

[2] S. Nudurupati, U.S. Bititci, V. Kumar, F.T.S. Chan., State of the art literature review on performance measurement, Computers \& Industrial Engineering, 60, (2): 279-290, 2011.

[3] S.K. Sikdar, Office of Research and Development, United States Environmental Protection Agency (EPA), Cincinnati, OH 45268 Perspective 1928 AIChE Journal, 49 (8). 2003.

[4] R.J.Singh, H.R. Murty, S.K. Gupta and A.K. Dikshit, An overview of sustainability assessment methodologies. Ecological Indicators, 9(2): 189-212, 2009.

[5] M. Imai, Kaizen: The Key to Japan's Competitive Success, McGraw Hill Higher Education, 260 p., 1986.

[6] A Smalley and I. Kato, Toyota Kaizen Methods: Six Steps to Improvement, Productivity Press, 156 p., 2010

[7] R. Shah and P.T. Ward, Lean manufacturing: context, practice bundles, and performance Journal of Operations Management, 21(2): 129149, 2003.
[8] J.P. Womack, D.T. Roos and D. Roos, The machine that changed the world, Free Press, New York, 339 p., 1990.

[9] T.T. Allen, Introduction to Engineering Statistics and Six SIGMA: Statistical Quality Control and Design of Experiments and Systems, Springer London Ltd, 2nd printing, 529 p., 2011.

[10] M.D. Mesarovic, D. Macko and Y. Takahara, Theory of Hierarchical Multilevel Systems, Academic Press, New York, 1970.

[11] V. Clivillé, L. Berrah and G. Mauris Quantitative expression and aggregation of performance measurements based on the MACBETH multicriteria method, International Journal of Production Economics, 105(1), 171-189, 2007.

[12] L. Berrah, G. Mauris and F. Vernadat, Information aggregation in industrial performance measurement: rationales, issues and definitions. International Journal of Production Research; 42(20): 4271-4293, 2004.

[13] M. Ehrgott and M. Wiecek, Multiobjective programming, in MCDA. Multiple Criteria Decision Analysis State of the Art Surveys. Kluwer Academic Publishers: 667-722, 2005.

[14]C. Labreuche and M. Grabisch, The Choquet integral for the aggregation of interval scales in multi-criteria decision-making, Fuzzy Sets and Systems, 137(1): 11-26, 2003.

[15] M. Grabisch, k-order additive discrete fuzzy measures and their representation, Fuzzy Sets and Systems, 92:167-189, 1997.

[16] L. Berrah, G. Mauris and J. Montmain, Monitoring the improvement of an overall industrial performance based on a Choquet integral aggregation, Omega, 36(3): 340-351, 2008.

[17] C.A. Bana e Costa and J.C. Vansnick, Applications of the MACBETH approach in the framework of an additive aggregation model, Journal of Multi-Criteria Decision Analysis, 6(2): 107-114, 1997.

[18] H. Mintzberg, D. Raisinghanin and A. Theoret, The structure of unstructured decision processes. Administrative Science Quaterly, 21:246-75, 1976.

[19] J. Lamont, Distributive justice, Stanford Encyclopedia of Philosophy, 8 pages, 2007.

[20]P. Slovic, L M. Finucane, E. Peters and D. G. MacGregor, The affect heuristic, European Journal of Operational Research, 177 (3): 1333 1352, 2007.

[21] A. Akharraz, G. Mauris, and J. Montmain, A project decision support system based on an elucidative fusion system. Fifth International Conference on Information Fusion (ICIF 02), Annapolis, USA, 593-599, July 2002. 